\title{
Construction of Orthogonal Complex Wavelets
}

\author{
Qingming Zhang \\ Tianjin University of Technology and Education No.1310,Dagu South Road, Hexi District, Tianjin 300222, China
}

\begin{abstract}
So for, the symmetric orthogonal complex wavelets with compact support were widely used in image processing and statistical model. In this paper, we present a construction method for parameterizing orthogonal complex wavelets. By this method, we can construct some complex wavelets with high sum rules or conjugate symmetric, at the same time, some examples are given in this paper.
\end{abstract}

Keywords: compact support; symmetry; orthogonality; complex wavelets; parameterization

\section{Introduction}

As is known to all, in addition to the Haar wavelet, any symmetric compactly supported orthogonal smooth real wavelet $^{[1-2]}$ do not exist. But in some applications, the symmetry is very necessary. In order to overcome the shortage with real wavelet and obtain this kind of wavelets, some researches are devoted to $\mathrm{M}(\mathrm{M}>2)$ scale wavelet, and higher dimensional wavelets and complex wavelets, etc. The complex wavelets were widely studied, and it has very good properties. With the orthogonal complex wavelets were extensively studied, Lawton ${ }^{[3]}$ constructed symmetric orthogonal complex wavelets, According to the orthogonality of Daubechies. Lina ${ }^{[4]}$ constructed symmetric orthogonal complex 2-wavelets. At present, based on a large number of references, there were constructed symmetric orthogonal complex wavelets. Whether can we find a way, which using the excellent characteristics of orthogonal complex wavelets to depict a variety of applications?

In this paper, inspired by Lai and Roach ${ }^{[5]}$, we presents a simple but effective parameterization method to determine the orthogonal complex wavelet filters. By choosing appropriate parameters, we can get some symmetry or high sum rules of orthogonal complex wavelets. Compared with the Lina's ${ }^{[4]}$ method, our method is more convenient.

Here is a need to use a few symbols and definitions in this paper. This paper $i$ is said the imaginary unit and $i^{2}=-1$. The Fourier transform of function $\mathrm{f}(\mathrm{x})$ is defined as:

$$
\hat{f}(\xi)=\int_{R} f(x) e^{-i \xi x} d x, f \in L_{1}(R)
$$

where one dimensional signal can be extended to two dimensional.

In general, constructing compactly supported wavelet corresponding scale function is defined as:

$$
\phi(t)=\sum_{k} p_{k} \phi(2 x-k)
$$

where the complex low-pass filter $p_{k}=x_{k}+i y_{k}$.

The corresponding mask symbol $P(\xi)$ can be defined as:

$$
P(\xi)=\frac{1}{2} \sum_{k} p_{k} e^{-i k \xi}
$$

Where $P(\xi)$ is limited nonzero trigonometric polynomial with complex coefficients.
If $\phi(t)$ are orthogonal, there are $P(0)=1$ and $|P(\xi)|^{2}+|P(\xi+\pi)|^{2}=1$; when the symbol satisfy the conjugate symmetry, there are $P(\xi)= \pm e^{-i c \xi} \bar{P}(-\xi)$ set up; when $P(\xi)$ satisfy the high sum rules, there are $P(\xi)=\left(1+e^{-i \xi}\right)^{m} Q(\xi)$ set up, where $Q(\xi)$ is some $2 \pi$-periodic trigonometric polynomial.

In this paper, the following are arranged as following: the second part, complex filter with length from four to five is given, and they satisfies the high sum rules or conjugate symmetric orthogonal complex wavelets required conditions. According to the different properties of the orthogonal complex wavelets, some examples are given. The last part concludes this paper.

\section{Main Results}

For positive integer $\mathrm{M}$, let

$$
P_{M}(\xi)=\frac{1}{2} \sum_{k=0}^{M-1} p_{k} e^{-i k \xi}
$$

where $P_{M}(\xi)$ is a trigonometric polynomial with a complex low-pass filter $\left\{p_{k}=x_{k}+i y_{k}\right\}_{k=0}^{M-1}, x_{k}, y_{k} \in R$ and $k=0,1, \cdots, M-1$.In the part, we present the construction of orthogonal complex wavelets method, where $M=4,5$.

Lemma $1^{[6]} P_{4}(\xi)$ satisfies $P_{4}(0)=1$ and

$\left|P_{4}(\xi)\right|^{2}+\left|P_{4}(\xi+\pi)\right|^{2}=1$, if and only if

$x_{0}=\frac{1}{2}+\frac{\sqrt{2}}{2} \cos \alpha \cos \beta, y_{0}=\frac{\sqrt{2}}{2} \sin \alpha \cos \gamma$,

$x_{1}=\frac{1}{2}+\frac{\sqrt{2}}{2} \cos \alpha \sin \beta, y_{1}=\frac{\sqrt{2}}{2} \sin \alpha \sin \gamma$,

$x_{2}=\frac{1}{2}-\frac{\sqrt{2}}{2} \cos \alpha \cos \beta, y_{2}=-\frac{\sqrt{2}}{2} \sin \alpha \cos \gamma$,

$x_{3}=\frac{1}{2}-\frac{\sqrt{2}}{2} \cos \alpha \sin \beta, y_{3}=-\frac{\sqrt{2}}{2} \sin \alpha \sin \gamma$

Where $\alpha, \beta, \gamma \in[0,2 \pi]$.

Theorem 1 Let $P_{4}(\xi)=\frac{1}{2} \sum_{k=0}^{3} p_{k} e^{-i k \xi},\left\{p_{k}=x_{k}+i y_{k}\right\}_{k=0}^{3}$ is orthogonal complex values scale filter with $\mathrm{M}=4$, and $P_{4}(\xi)=\left(1+e^{-i \xi}\right)^{2}\left(a e^{-i \xi}+b\right)$, if and only if 


\section{International Journal of Science and Research (IJSR)

$a=\frac{1}{4}-\frac{\sqrt{2}}{4} \cos \alpha \sin \beta-i \frac{\sqrt{2}}{2} \sin \alpha \sin \gamma$

$b=\frac{1}{4}+\frac{\sqrt{2}}{4} \cos \alpha \cos \beta+i \frac{\sqrt{2}}{2} \sin \alpha \cos \gamma$

Where $\alpha, \beta, \lambda$ satisfies $\left\{\begin{array}{c}\sqrt{2} \cos \alpha(\sin \beta-\cos \beta)=1 \\ \gamma=\frac{\pi}{4}, \frac{5 \pi}{4}\end{array}\right.$

Proof: By

$$
\begin{aligned}
& P_{4}(\xi)=\left(1+e^{-i \xi}\right)^{2}\left(a e^{-i \xi}+b\right) \\
& =b+(a+b) e^{-i \xi}+(2 a+b) e^{-i 2 \xi}+a e^{-i 3 \xi}
\end{aligned}
$$

and

$P_{4}(\xi)=\frac{1}{2} \sum_{k=0}^{3} p_{k} e^{-i k \xi}=\frac{1}{2} p_{0}+\frac{1}{2} p_{1} e^{-i \xi}+\frac{1}{2} p_{2} e^{-i 2 \xi}+\frac{1}{2} p_{3} e^{-i 3 \xi}$

Then we have $\quad\left\{\begin{array}{c}p_{1}=p_{3}+2 p_{0} \\ p_{2}=2 p_{3}+p_{0} \\ a=\frac{1}{2} p_{3} \\ b=\frac{1}{2} p_{0}\end{array}\right.$

and $\left\{\begin{array}{l}x_{1}=x_{3}+2 x_{0} \\ y_{1}=y_{3}+2 y_{0} \\ x_{2}=2 x_{3}+x_{0} \\ y_{2}=2 y_{3}+y_{0}\end{array}\right.$

From (1), we can solution $\left\{\begin{aligned} \sqrt{2} \cos \alpha(\sin \beta-\cos \beta)=1 \\ \gamma=\frac{\pi}{4}, \frac{5 \pi}{4} \\ a=\frac{1}{2} p_{3} \\ b=\frac{1}{2} p_{0}\end{aligned}\right.$

Example 1 Let $\alpha=\frac{2 \pi}{3}, \beta=\frac{7 \pi}{4}, \gamma=\frac{\pi}{4}$, then

$$
P_{4}(\xi)=\frac{\sqrt{3}}{3}\left(\frac{1+e^{-i \xi}}{2}\right)^{2}\left(\sqrt{3}\left(1+e^{-i \xi}\right)+3\left(1-e^{-i \xi}\right) i\right)
$$

Example 2 Let $\alpha=\frac{\pi}{4}, \beta=\frac{\pi}{2}, \gamma=\frac{\pi}{4}$, then

$$
\begin{gathered}
x_{0}=\frac{1}{4}-\frac{\sqrt{2}}{4} \cos \alpha \cos \beta+\frac{C}{2} \cos \eta, y_{0}=-\frac{\sqrt{2}}{4} \sin \alpha \cos \gamma+\frac{C}{2} \sin \eta, \\
x_{2}=\frac{1}{2}+\frac{\sqrt{2}}{2} \cos \alpha \cos \beta, y_{2}=\frac{\sqrt{2}}{2} \sin \alpha \cos \gamma, \\
x_{4}=\frac{1}{4}-\frac{\sqrt{2}}{4} \cos \alpha \cos \beta-\frac{C}{2} \cos \eta, y_{4}=-\frac{\sqrt{2}}{4} \sin \alpha \cos \gamma-\frac{\sqrt{2}}{2} \sin \eta,
\end{gathered}
$$

Where $C=\frac{1}{2} \sqrt{(1-\sqrt{2} \cos \alpha \cos \beta)^{2}+2 \cos ^{2} \gamma \sin ^{2} \alpha}$,

$\alpha, \beta, \gamma, \eta \in[0,2 \pi]$

Theorem 3 Let $P_{5}(\xi)=\frac{1}{2} \sum_{k=0}^{4} p_{k} e^{-i k \xi}, \quad\left\{p_{k}=x_{k}+i y_{k}\right\}_{k=0}^{4}$ is orthogonal complex values scale filter with $\mathrm{M}=5$, and $P_{5}(\xi)=\left(1+e^{-i \xi}\right)^{2}\left(a e^{-i 2 \xi}+b e^{-i \xi}+c\right)$, if and only if

$$
P_{4}(\xi)=\frac{\sqrt{2}}{2}\left(\frac{1+e^{-i \xi}}{2}\right)^{2}\left(\left(1-e^{-i \xi}\right) i\right)
$$

Theorem 2 Let $P_{4}(\xi)=\frac{1}{2} \sum_{k=0}^{3} p_{k} e^{-i k \xi}, \quad\left\{p_{k}=x_{k}+i y_{k}\right\}_{k=0}^{3}$ is orthogonal complex values scale filter with $\mathrm{M}=4$, and $P_{4}(\xi)=e^{-i 3 \xi} \overline{P_{4}}(-\xi)$, then

$$
\left\{\begin{array}{l}
\alpha \in[0,2 \pi] \\
\beta=\frac{3 \pi}{4}, \frac{7 \pi}{4} \\
\gamma=\frac{\pi}{4}, \frac{5 \pi}{4}
\end{array}\right.
$$

Proof: By $P_{4}(\xi)=e^{-i 3 \xi} \overline{P_{4}}(-\xi)$ and $P_{4}(\xi)=\frac{1}{2} \sum_{k=0}^{3} p_{k} e^{-i k \xi}$, then we have

$$
\begin{aligned}
& p_{0}+p_{1} e^{-i \xi}+p_{2} e^{-i 2 \xi}+p_{3} e^{-i 3 \xi} \\
& =e^{-i 3 \xi}\left(\overline{p_{0}}+\overline{p_{1}} e^{i \xi}+\overline{p_{2}} e^{i 2 \xi}+\overline{p_{3}} e^{i 3 \xi}\right) \\
& =\overline{p_{0}} e^{-i 3 \xi}+\overline{p_{1}} e^{-i 2 \xi}+\overline{p_{2}} e^{-i \xi}+\overline{p_{3}}
\end{aligned}
$$

So we have $\left\{\begin{array}{l}p_{0}=\overline{p_{3}} \\ p_{1}=\overline{p_{2}}\end{array}\right.$ or $\left\{\begin{array}{c}x_{0}=x_{3} \\ y_{0}=-y_{3} \\ x_{1}=x_{2} \\ y_{1}=-y_{2}\end{array}\right.$

$$
\text { From (1), we can obtain solution }\left\{\begin{array}{l}
\alpha \in[0,2 \pi] \\
\beta=\frac{3 \pi}{4}, \frac{7 \pi}{4} \\
\gamma=\frac{\pi}{4}, \frac{5 \pi}{4}
\end{array}\right.
$$

Example 3 Let $\alpha=\frac{\pi}{2}, \beta=\frac{3 \pi}{4}, \gamma=\frac{\pi}{4}$, then

$$
P_{4}(\xi)=\frac{1}{4}\left[(1+i)+(1+i) e^{-i \xi}+(1-i) e^{-i 2 \xi}+(1-i) e^{-i 3 \xi}\right]
$$

Lemma $2^{[6]} \quad P_{5}(\xi)$ satisfies $P_{5}(0)=1$ and $\left|P_{5}(\xi)\right|^{2}+\left|P_{5}(\xi+\pi)\right|^{2}=1$, if and only if

\section{Volume 5 Issue 4, April 2016}




$$
\left\{\begin{array}{c}
a=\frac{1}{8}-\frac{\sqrt{2}}{8} \cos \alpha \cos \beta-\frac{C}{4} \cos \eta \\
+i\left(-\frac{\sqrt{2}}{8} \sin \alpha \cos \gamma-\frac{C}{4} \sin \eta\right) \\
b=\frac{\sqrt{2}}{4} \cos \alpha \sin \beta+\frac{\sqrt{2}}{4} \cos \alpha \cos \beta-\frac{C}{2} \cos \eta \\
+i\left(\frac{\sqrt{2}}{4} \sin \alpha \sin \gamma+\frac{\sqrt{2}}{4} \sin \alpha \cos \gamma-\frac{C}{2} \sin \eta\right) \\
c=\frac{1}{8}-\frac{\sqrt{2}}{8} \cos \alpha \cos \beta+\frac{C}{4} \cos \eta \\
+i\left(-\frac{\sqrt{2}}{8} \sin \alpha \cos \gamma+\frac{C}{4} \sin \eta\right)
\end{array}\right.
$$

where $\alpha, \beta, \lambda, \eta, C$ satisfies

$$
\left\{\begin{array}{c}
\frac{\sqrt{2}}{2} \cos \alpha \sin \beta=\frac{3 C}{4} \cos \eta \\
\frac{\sqrt{2}}{2} \sin \alpha \sin \gamma=C \sin \eta \\
C=\frac{1}{2} \sqrt{(1-\sqrt{2} \cos \alpha \cos \beta)^{2}+2 \cos ^{2} \gamma \sin ^{2} \alpha}
\end{array}\right.
$$

Proof: By

$$
\begin{aligned}
& P_{5}(\xi)=\left(1+e^{-i \xi}\right)^{2}\left(a e^{-i 2 \xi}+b e^{-i \xi}+c\right) \\
& =c+(b+2 c) e^{-i \xi}+(a+2 b+c) e^{-i 2 \xi}+(2 a+b) e^{-i 3 \xi}+a e^{-i 4 \xi}
\end{aligned}
$$

and

$$
P_{5}(\xi)=\frac{1}{2} \sum_{k=0}^{4} p_{k} e^{-i k \xi}=\frac{1}{2} p_{0}+\frac{1}{2} p_{1} e^{-i \xi}+\frac{1}{2} p_{2} e^{-i 2 \xi}+\frac{1}{2} p_{3} e^{-i 3 \xi}+\frac{1}{2} p_{4} e^{-i 4 \xi}
$$

$$
\text { Then we have }\left\{\begin{array}{c}
\frac{1}{2} p_{0}=c \\
\frac{1}{2} p_{1}=b+2 c \\
\frac{1}{2} p_{2}=a+2 b+c \\
\frac{1}{2} p_{3}=2 a+b \\
\frac{1}{2} p_{4}=a
\end{array}\right.
$$

From (2), we can solution

$$
\left\{\begin{array}{c}
a=\frac{1}{8}-\frac{\sqrt{2}}{8} \cos \alpha \cos \beta-\frac{C}{4} \cos \eta \\
+i\left(-\frac{\sqrt{2}}{8} \sin \alpha \cos \gamma-\frac{C}{4} \sin \eta\right) \\
b=\frac{\sqrt{2}}{4} \cos \alpha \sin \beta+\frac{\sqrt{2}}{4} \cos \alpha \cos \beta-\frac{C}{2} \cos \eta \\
+i\left(\frac{\sqrt{2}}{4} \sin \alpha \sin \gamma+\frac{\sqrt{2}}{4} \sin \alpha \cos \gamma-\frac{C}{2} \sin \eta\right) \\
c=\frac{1}{8}-\frac{\sqrt{2}}{8} \cos \alpha \cos \beta+\frac{C}{4} \cos \eta \\
+i\left(-\frac{\sqrt{2}}{8} \sin \alpha \cos \gamma+\frac{C}{4} \sin \eta\right)
\end{array}\right.
$$

Where $\alpha, \beta, \lambda, \eta, C$ satisfies

$$
\left\{\begin{array}{c}
\frac{\sqrt{2}}{2} \cos \alpha \sin \beta=\frac{3 C}{4} \cos \eta \\
\frac{\sqrt{2}}{2} \sin \alpha \sin \gamma=C \sin \eta \\
C=\frac{1}{2} \sqrt{(1-\sqrt{2} \cos \alpha \cos \beta)^{2}+2 \cos ^{2} \gamma \sin ^{2} \alpha}
\end{array}\right.
$$

Example 4 Let $\alpha=\arccos \left(\frac{\sqrt{6}+\sqrt{2}}{4}\right), \beta=0, \gamma=\frac{\pi}{2}, \eta=\frac{\pi}{2}$, then

$$
P_{5}(\xi)=\frac{\sqrt{3}-1}{2}\left(\frac{1+e^{-i \xi}}{2}\right)^{2}\left((-1+i)+(4+2 \sqrt{3}) e^{-i \xi}-(1+i) e^{-i 2 \xi}\right)
$$

Example 5 Let $\alpha=\frac{\pi}{2}, \beta=0, \gamma=\frac{\pi}{3}, \eta=\frac{\pi}{2}$, then

$$
P_{5}(\xi)=\frac{\sqrt{2}}{4}\left(\frac{1+e^{-i \xi}}{2}\right)^{2}\left(2 \sqrt{2}+(\sqrt{3}-1) i+2 i e^{-i \xi}-(1+\sqrt{3}) i e^{-i 2 \xi}\right)
$$

Theorem 4 Let $P_{5}(\xi)=\frac{1}{2} \sum_{k=0}^{4} p_{k} e^{-i k \xi}, \quad\left\{p_{k}=x_{k}+i y_{k}\right\}_{k=0}^{4}$ is orthogonal complex values scale filter with $\mathrm{M}=5$, and $P_{5}(\xi)=\left(1+e^{-i \xi}\right)^{3}\left(a e^{-i \xi}+b\right)$, if and only if

$$
\left\{\begin{array}{l}
a=\frac{1}{8}-\frac{\sqrt{2}}{8} \cos \alpha \cos \beta-\frac{C}{4} \cos \eta \\
+i\left(-\frac{\sqrt{2}}{8} \sin \alpha \cos \gamma-\frac{C}{4} \sin \eta\right) \\
b=\frac{1}{8}-\frac{\sqrt{2}}{8} \cos \alpha \cos \beta+\frac{C}{4} \cos \eta \\
+i\left(-\frac{\sqrt{2}}{8} \sin \alpha \cos \gamma+\frac{C}{4} \sin \eta\right)
\end{array}\right.
$$

Where $\alpha, \beta, \lambda, \eta, C$ satisfies

$$
\left\{\begin{array}{c}
\frac{\sqrt{2}}{2} \cos \alpha \sin \beta=\frac{1}{2}-\sqrt{2} \cos \alpha \cos \beta+C \cos \eta \\
\frac{\sqrt{2}}{2} \sin \alpha \sin \gamma=-\sqrt{2} \sin \alpha \cos \gamma+C \sin \eta \\
C=\frac{1}{2} \sqrt{(1-\sqrt{2} \cos \alpha \cos \beta)^{2}+2 \cos ^{2} \gamma \sin ^{2} \alpha}
\end{array}\right.
$$

Proof: By

$$
\begin{aligned}
& P_{5}(\xi)=\left(1+e^{-i \xi}\right)^{3}\left(a e^{-i \xi}+b\right) \\
& =b+(a+3 b) e^{-i \xi}+(3 a+3 b) e^{-i 2 \xi}+(3 a+b) e^{-i 3 \xi}+a e^{-i 4 \xi} \\
& \quad \text { and } \\
& P_{5}(\xi)=\frac{1}{2} \sum_{k=0}^{4} p_{k} e^{-i k \xi}=\frac{1}{2} p_{0}+\frac{1}{2} p_{1} e^{-i \xi}+\frac{1}{2} p_{2} e^{-i 2 \xi}+\frac{1}{2} p_{3} e^{-i 3 \xi}+\frac{1}{2} p_{4} e^{-i 4 \xi}
\end{aligned}
$$




$$
\begin{aligned}
& \text { Then we have }\left\{\begin{array}{l}
\frac{1}{2} p_{0}=b \\
\frac{1}{2} p_{1}=a+3 b \\
\frac{1}{2} p_{2}=3 a+3 b \\
\frac{1}{2} p_{3}=3 a+b \\
\frac{1}{2} p_{4}=a
\end{array},\right. \text { from (2), we can solution } \\
& \left\{\begin{array}{l}
a=\frac{1}{8}-\frac{\sqrt{2}}{8} \cos \alpha \cos \beta-\frac{C}{4} \cos \eta \\
+i\left(-\frac{\sqrt{2}}{8} \sin \alpha \cos \gamma-\frac{C}{4} \sin \eta\right. \\
b=\frac{1}{8}-\frac{\sqrt{2}}{8} \cos \alpha \cos \beta+\frac{C}{4} \cos \eta \\
+i\left(-\frac{\sqrt{2}}{8} \sin \alpha \cos \gamma+\frac{C}{4} \sin \eta\right)
\end{array}\right. \\
& \text { Where } \alpha, \beta, \lambda, \eta, C \operatorname{satisfies} \\
& \begin{array}{c}
\frac{\sqrt{2}}{2} \cos \alpha \sin \beta=\frac{1}{2}-\sqrt{2} \cos \alpha \cos \beta+C \cos \eta \\
\frac{\sqrt{2}}{2} \sin \alpha \sin \gamma=-\sqrt{2} \sin \alpha \cos \gamma+C \sin \eta \\
\frac{1}{2} \sqrt{(1-\sqrt{2} \cos \alpha \cos \beta)^{2}+2 \cos { }^{2} \gamma \sin { }^{2} \alpha}
\end{array}
\end{aligned}
$$

Theorem 5 Let $P_{5}(\xi)=\frac{1}{2} \sum_{k=0}^{4} p_{k} e^{-i k_{\xi}^{\xi}}, \quad\left\{p_{k}=x_{k}+i y_{k}\right\}_{k=0}^{4}$ is orthogonal complex values scale filter with $\mathrm{M}=4$, and $P_{5}(\xi)=e^{-i 4 \xi} \overline{P_{4}}(-\xi)$, then

$$
\left\{\begin{array}{c}
C \cos \eta=0 \\
\sin \alpha \cos \gamma=0 \\
\cos \alpha \sin \beta=0 \\
C=\frac{1}{2} \sqrt{(1-\sqrt{2} \cos \alpha \cos \beta)^{2}+2 \cos ^{2} \gamma \cos ^{2} \alpha}
\end{array}\right.
$$

Proof: By

$P_{5}(\xi)=e^{-i 4 \xi} \overline{P_{5}}(-\xi)$ and $P_{5}(\xi)=\frac{1}{2} \sum_{k=0}^{4} p_{k} e^{-i k \xi}$, then we

have

$$
\begin{aligned}
& p_{0}+p_{1} e^{-i \xi}+p_{2} e^{-i 2 \xi}+p_{3} e^{-i 3 \xi}+p_{4} e^{-i 4 \xi} \\
& =e^{-i 4 \xi}\left(\overline{p_{0}}+\overline{p_{1}} e^{i \xi}+\overline{p_{2}} e^{i 2 \xi}+\overline{p_{3}} e^{i 3 \xi}+\overline{p_{4}} e^{i 4 \xi}\right) \\
& =\overline{p_{0}} e^{-i 4 \xi}+\overline{p_{1}} e^{-i 3 \xi}+\overline{p_{2}} e^{-i 2 \xi}+\overline{p_{3}} e^{-i \xi}+\overline{p_{4}} \\
& \text { so }\left\{\begin{array} { l } 
{ p _ { 0 } = \overline { p _ { 4 } } } \\
{ p _ { 1 } = \overline { p _ { 3 } } } \\
{ p _ { 2 } = \overline { p _ { 2 } } }
\end{array} \text { or } \left\{\begin{array}{c}
x_{0}=x_{4} \\
y_{0}=-y_{4} \\
x_{1}=x_{3} \\
y_{1}=-y_{3} \\
y_{2}=-y_{2}
\end{array}\right.\right.
\end{aligned}
$$

From (2), we can solution

$$
\left\{\begin{array}{c}
C \cos \eta=0 \\
\sin \alpha \cos \gamma=0 \\
\cos \alpha \sin \beta=0 \\
C=\frac{1}{2} \sqrt{(1-\sqrt{2} \cos \alpha \cos \beta)^{2}+2 \cos ^{2} \gamma \sin ^{2} \alpha}
\end{array}\right.
$$

Example 6 Let $\alpha=\frac{\pi}{2}, \beta=0, \gamma=\frac{\pi}{2}, \eta=\frac{\pi}{2}$, then

$$
P_{5}(\xi)=\frac{1}{8}\left[(1+i)+(2+2 \sqrt{2}) i e^{-i \xi}+2 e^{-i 2 \xi}+(2-2 \sqrt{2}) i e^{-i 3 \xi}+(1-i) e^{-i 4 \xi}\right]
$$

\section{Conclusion}

At present, the symmetric orthogonal compactly supported complex wavelets were widely applied to image processing and statistical model, based on orthogonal wavelet necessary condition. In this paper, we present a construction method for parameterizing orthogonal complex wavelets. By this method, we can construct some complex wavelets with high sum rules or conjugate symmetric, at the same time, some examples are given. In addition, with the increase of positive integer $\mathrm{M}$, the number of parameters is increased.

\section{References}

[1] Tang Xiaochu. Wavelet analysis and its application [M]. Chongqing: Chongqing publishing house, 2006.

[2] FERNANDES Felix C A, IVAN W Selesnick, Van RUTGER L C Spaendonck . Complex wavelet transforms with allpass filters [J. Signal Processing, 2003, 83( 8) : 1 689-1 706

[3] Lawton W. Applications of complex valued wavelet transforms to subband decomposition[J]. IEEE Transaction on Signal Process, 1993,44(12):3566-3568

[4] Lina J M. Complex daubechies wavelets[J]. Applied and Computational Harmonic Analysis, 1995,2(3):219-229

[5] Lai M J, Roach D W. Parameterization of univariate orthogonal wavelets with short support [C] // Approximation theory X: Wavelets, Splines, and Applications, Nashville, Tenn, USA, Vanderbilt University Press, 2002:369-384

[6] FENG Yan, YUAN De-hui, YANG Shou-zhi, Parameterizations of Orthogonal Complex Wavelets with Short Support, CHINESE JOURNAL OF ENGINEERING MATHEMATICS, 2013,5(17):773-780

\section{Author Profile}

Qingming Zhang is reading the M.S. degrees in College of Science from Tianjin University of Technology and Education . 